> Les vésicules extracellulaires (VE) correspondent à un ensemble hétérogène de nanovésicules membranaires sécrétées dans le milieu extracellulaire et circulant dans les différents fluides de l'organisme. Ces VE véhiculent du matériel biologique (protéines, lipides, acides nucléiques) qu'elles peuvent transférer à des cellules/tissus cibles, modulant ainsi leur réponse et/ou leur phénotype. Les dysfonctions caractérisant les maladies métaboliques liées à l'obésité sont associées à des modifications des concentrations circulantes de $V \varepsilon$ ainsi qu'à des altérations de leur contenu. L'intérêt grandissant porté aux VE comme nouveaux vecteurs de communication intercellulaire a conduit à s'interroger sur leur rôle dans le développement des complications métaboliques. Dans cette synthèse, nous résumerons la littérature portant sur les VE circulantes comme potentiels marqueurs des maladies métaboliques. Nous détaillerons ensuite le dialogue vésiculaire inter-organes responsable du développement des complications associées à l'obésité. Enfin, nous discuterons les futures pistes de recherche qui contribueront à mieux appréhender le lien entre $V \varepsilon$ et maladies métaboliques. <

La coexistence de plusieurs troubles physiologiques d'origine lipidique, glucidique ou vasculaire, associés à un excès de poids chez un même individu, définit le syndrome métabolique (SMet) [1]. L'association de ces facteurs de risque augmente considérablement le risque de maladies cardiométaboliques, à l'instar du diabète de type 2 (DT2), de maladies cardiovasculaires et hépatiques, de troubles respiratoires (apnées du sommeil) ou encore de cancers. L'obésité viscérale est considérée comme un des facteurs les plus délétères dans le développement des maladies métaboliques et est souvent

Vignette (๔ Guillaume van Niel, Aurélie di Cicco, Graça Raposo, Daniel Levy).

\section{Vésicules extracellulaires et maladies métaboliques}

\section{Des liaisons dangereuses}

Alexia Blandin ${ }^{1,2}$, Soazig Le Lay ${ }^{1,2}$

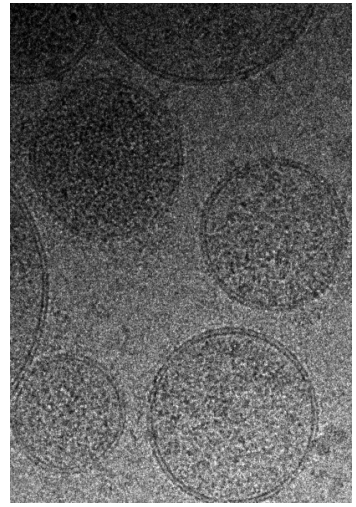

${ }^{1}$ Université de Nantes, CNRS, Inserm, Institut du thorax, F-44000 Nantes, France. ${ }^{2}$ Univ Angers, SFR Interactions cellulaires et applications thérapeutiques (ICAT), F-49000 Angers, France. soazig.lelay@inserm.fr

associée à une inflammation chronique et générale dite de bas-grade. L'évaluation des différents critères métaboliques définissant le SMet (tour de taille, glycémie à jeun, triglycérides, lipoprotéines de haute densité [HDL]-cholestérol, pression artérielle), associée au dosage de cytokines/adipokines, est utilisée en pratique clinique pour définir le profil métabolique du patient, mais n'est pas informative sur les atteintes métaboliques respectives des différents organes. Dans ce contexte, les vésicules extracellulaires ( $V \varepsilon)$, dont le contenu reflète l'état physiopathologique des cellules qui les sécrètent, apparaissent comme des biomarqueurs et/ou des médiateurs des complications métaboliques.

Les $V \varepsilon$ constituent un ensemble hétérogène de nanovésicules membranaires sécrétées dans le milieu extracellulaire et circulant dans les différents fluides de l'organisme (sang, urine, lymphe, etc.). Elles regroupent, d'une part, des vésicules de grande taille (gVE, $100 \mathrm{~nm}-1$ $\mu \mathrm{m}$ ), libérées par bourgeonnement de la membrane plasmique, et, d'autre part, des vésicules de petite taille ( $p V \varepsilon,<100 \mathrm{~nm}$ ) correspondant à la sécrétion par exocytose des vésicules intra-luminales des corps multivésiculaires [2]. Ces VE véhiculent du matériel biologique (protéines, lipides, acides nucléiques) qu'elles peuvent transférer à des cellules/tissus cibles. Dès les années 1990, la cytométrie en flux a permis de déterminer l'origine cellulaire des gV circulantes et d'établir que les plaquettes sanguines étaient la source cellulaire principale de ces gVE circulantes à côté des gVE d'origine monocytaire, lymphocytaire, érythrocytaire ou endothéliale (les gVE plaquettaires représentant, selon les études, entre 50 et $90 \%$ des gVE circulantes). Les concentrations circulantes de gVE totales, et de certaines souspopulations de gVE ont ainsi été largement étudiées dans les maladies 


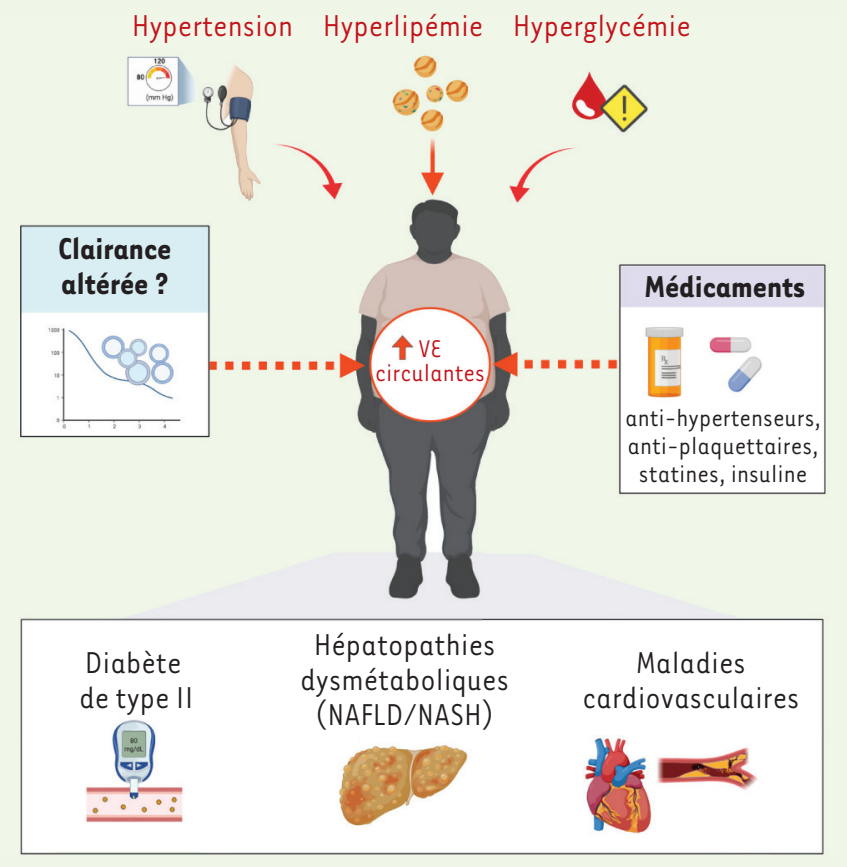

Figure 1. Les maladies métaboliques sont associées à une augmentation des concentrations de vésicules extracellulaires (VE) circulantes. Les facteurs de risque associés au développement des maladies métaboliques stimulent la sécrétion des $V \mathcal{E}$, contribuant à augmenter leurs concentrations circulantes. La prise de médicaments ou encore une clairance altérée pourraient également participer à maintenir des concentrations plasmatiques élevées de $V \varepsilon$.

cardiovasculaires [3]. L'intérêt porté à ces vésicules s'est élargi ces dernières années aux pVE dans le contexte des maladies métaboliques.

\section{Les $V \varepsilon$ circulantes : des biomarqueurs des maladies métaboliques?}

Les $V \varepsilon$ constituent de potentiels biomarqueurs de l'état métabolique du patient, ce qui a conduit à les quantifier et à les caractériser dans des biofluides facilement accessibles, tels que le sang ou les urines, chez des patients à risque ou souffrant de maladies métaboliques.

\section{Les maladies métaboliques sont associées à de fortes} concentrations de $V \varepsilon$ circulantes

Les concentrations de $p V \varepsilon$ et de gVE plasmatiques sont significativement augmentées chez les patients souffrant de SMet $[4,5]$, et sont corrélées à l'indice de masse corporelle (IMC), soulignant l'importance de l'obésité [6] (Figure 1). Cette augmentation se retrouve également chez des patients hypertendus présentant une hyperlipémie ou souffrant de maladies athéro-thrombotiques [3], chez des patients diabétiques de type 2 (DT2) [7], et chez des patients souffrant d'hépatopathies dysmétaboliques [8]. La réduction des concentrations de VE plasmatiques observée lors d'une perte de poids, suite à une restriction alimentaire ou à une chirurgie bariatrique, suggère que les VE adipocytaires pourraient participer à cette élévation [9]. Cependant, l'absence de marqueurs spécifiques permettant l'identification des $V \varepsilon$ sécrétées par les adipocytes ne permet pas la quantification des $V \varepsilon$ adipocytaires circulantes. En revanche, l'élévation des $V \varepsilon$ plasmatiques observée dans les complications métaboliques associées à l'obésité s'accompagne généralement d'une élévation concomitante des $V \varepsilon$ d'origine plaquettaire, érythrocytaire, leucocytaire ou endothéliale $[3,6,7]$.

Les niveaux accrus de $V \varepsilon$ plasmatiques peuvent résulter de l'activation ou de l'apoptose de différentes cellules vasculaires à la suite d'un stimulus pathologique, bien qu'une clairance altérée de ces $V \varepsilon$ causée par différents facteurs de risque cardiovasculaire ne puisse être exclue [3]. L'insulino-résistance (IR) et l'hyperglycémie associées induisent également la sécrétion de $V \varepsilon$ contribuant à maintenir de fortes concentrations de VE circulantes chez les patients diabétiques [10]. Enfin, les médicaments (anti-plaquettaires, anti-hypertenseurs, statines ou insuline) bien souvent prescrits aux patients présentant un risque cardiovasculaire élevé, influencent également les niveaux de $V \varepsilon$ circulantes et/ ou leur contenu [3]. II est difficile de distinguer l'effet direct de ces médicaments sur la sécrétion cellulaire des $V \varepsilon$ et leur bénéfice sur I'hypertension ou la dyslipidémie, paramètres métaboliques modulant également la composition et la concentration des VE.

\section{Les vésicules extracellulaires circulantes: de}

nouveaux biomarqueurs des maladies métaboliques? Les $V \varepsilon$ sécrétées dans le milieu extracellulaire transportent des composants cellulaires provenant des cellules dont elles sont issues. Leur caractérisation peut ainsi avoir une valeur diagnostique et/ou pronostique pour certaines maladies métaboliques.

Plusieurs études ont ainsi démontré un fort taux circulant de gVE plaquettaires ou endothéliales chez des patients souffrant d'athérosclérose [11], tandis que des niveaux circulants élevés de gVE leucocytaires sont associés à des plaques athérosclérotiques instables [12]. Les gVE d'origine endothéliale améliorent le potentiel prédictif pour identifier les patients à risque de survenue d'évènements cardiovasculaires [13]. À ce jour, la caractérisation des pVE dans les maladies cardiovasculaires reste limitée, principalement en raison des difficultés à les quantifier par cytométrie en flux et à les séparer des lipoprotéines plasmatiques avec lesquelles elles partagent des caractéristiques physicochimiques.

Le contenu vésiculaire peut également être informatif de l'évolution des maladies métaboliques. Ainsi, la 


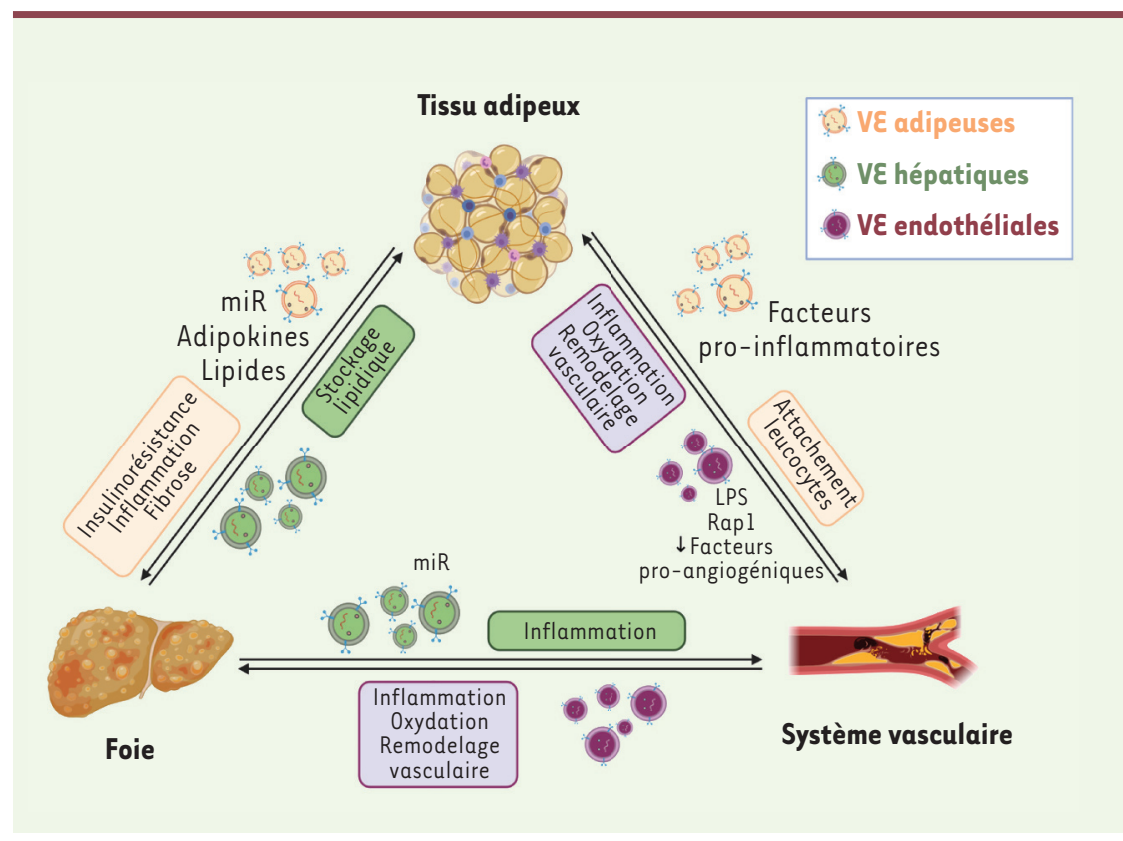

Figure 2. Le dialogue vésiculaire inter-organes contribue à la mise en place des dysfonctions métaboliques. Dans un contexte d'obésité et de fort risque cardiométabolique, différents organes participant au métabolisme sécrètent des $V \varepsilon$. Les cargos qu'elles transportent, de natures protéique, nucléique ou lipidique, reflètent l'état physiopathologique de l'organisme. Leur transfert à des cellules/tissus cibles conduisent à des réponses délétères participant à la mise en place des dysfonctions métaboliques.

présence d'aquaporines, protéines des canaux hydriques, dans les $p V \varepsilon$ urinaires, constitue un biomarqueur prometteur pour le diagnostic de cancers rénaux ou, plus largement, de maladies rénales [14]. Le contenu des $V \varepsilon$ en matériel génétique, à l'image des nombreux petits ARN (micro-ARN ou miR) qu'elles transportent, est aussi modulé par les différents facteurs de risque métabolique. Ainsi, les pVE plasmatiques de patients obèses ou insulino-résistants sont enrichies en miR capables de réguler le contenu lipidique ou la signalisation insulinique des adipocytes [15]. Le criblage de certains miR vésiculaires, ou plus largement du contenu des $V E$, pourrait ainsi être utilisé à des fins diagnostiques. Plusieurs miR ou protéines, enrichis dans les VE urinaires ou sanguines de patients souffrant de maladies hépatiques, représentent ainsi des candidats potentiels [8]. Cette perspective représente un intérêt majeur pour les maladies hépatiques pour lesquelles I'utilisation des VE comme outil diagnostic renseignerait sur l'évolution de la maladie, remplaçant la biopsie de foie, plus invasive.

\section{Les vésicules extracellulaires (ve), actrices du dialogue inter- organe dans les maladies métaboliques?}

\section{Les $V \varepsilon$ et le diabète de type 2}

L'obésité est généralement associée au développement d'une résistance à l'insuline, qui précède souvent l'apparition d'un diabète de type 2 (DT2). Cette résistance à l'hormone se traduit par une réduction de ses effets métaboliques, c'est-à-dire un défaut d'entrée de glucose et une moindre inhibition de la lipolyse dans les tissus insulino-dépen- dants, l'ensemble favorisant une hyperlipémie. Le tissu adipeux n'assumant plus son rôle de stockage, les lipides se déposent alors de manière ectopique dans le foie, le muscle squelettique, le cœur ou dans les vaisseaux, ce qui conduit à des phénomènes de lipotoxicité.

Les adipocytes sécrètent d'importantes quantités de gVE et de $p V \varepsilon$ qui présentent des signatures protéiques et lipidiques distinctes, présageant d'effets métaboliques variés [16]. Les stimulus environnementaux associés à l'obésité, comme l'afflux massif de lipides, I'hypoxie ou l'inflammation, stimulent la sécrétion de ces $V \varepsilon$ adipocytaires, conduisant à leur augmentation chez les sujets obèses $[16,17]$. En outre, les VE sécrétées par la fraction stroma-vasculaire du tissu adipeux (composée de préadipocytes, de cellules endothéliales, de cellules immunitaires, etc..) participent également à l'important trafic vésiculaire existant au sein de ce tissu. Ce trafic dépendant des $V \varepsilon$ participe ainsi à l'homéostasie du tissu adipeux, à sa vascularisation, sa capacité de stockage lipidique ou à son inflammation [17].

L'injection de $p V E$ dérivées d'explants de tissu adipeux de souris obèses est suffisante pour instaurer une insulinorésistance chez des souris saines, démontrant le potentiel de ces $V \varepsilon$ adipeuses à reproduire les effets délétères du sécrétome adipeux de souris obèses [18]. La résistance induite est couplée à l'activation de macrophages adipeux résidents et leur orientation vers un profil pro-inflammatoire (de type Ml) [18]. Elle peut également être induite par l'injection de VE dérivées uniquement des macrophages de tissus adipeux obèses [19]. L'invalidation du gène codant le récepteur de l'immunité innée TLR4 (Toll-like receptor 4) suffit à contrer les effets délétères des pVE de tissus adipeux obèses, suggérant que l'insulinorésistance induite par ces $p V \varepsilon$ est fortement liée à une réponse inflammatoire dépendante de l'immunité innée.

Plusieurs composants véhiculés par les VE adipeuses pourraient être impliqués dans ces effets délétères (Figure 2). Le tissu adipeux constitue une source importante de miR véhiculés par les $V \varepsilon$, parmi lesquels certains régulent l'expression de protéines clés de la signalisation insulinique et/ou l'activation de voies de signalisation pro-inflammatoires et pro-fibrotiques 
dans des organes cibles, dont le foie [19]. Plusieurs adipokines, aux propriétés pro-inflammatoires, pro-fibrosantes ou régulant le métabolisme glucido-lipidique, sont également des cargos protéiques des VE adipeuses [6, 20]. Enfin, l'afflux massif de palmitate, fortement enrichi dans notre alimentation actuelle, stimule la sécrétion de $V \varepsilon$ adipocytaires, hépatocytaires ou musculaires $[16,17]$. Ces VE se retrouvent enrichies en lipides lipotoxiques qui altèrent ainsi la sensibilité à l'insuline des cellules cibles [21].

L'ensemble de ces données soulignent l'importance des $V \varepsilon$, notamment celles sécrétées par le tissu adipeux dans un contexte d'obésité, dans le développement du DT2, complication métabolique considérée comme initiatrice des autres dysfonctions métaboliques associées à l'obésité.

\section{VE, maladies métaboliques et maladies cardiovasculaires}

La dysfonction endothéliale est une altération vasculaire précoce qui favorise la survenue de maladies cardiovasculaires. Elle apparaît souvent suite à une résistance à l'insuline. La communication entre les cellules endothéliales et leur microenvironnement tissulaire contribue à la libération de signaux pour attirer les cellules immunitaires et les capter à partir de la circulation, ce qui favorise le développement de plaques athéromateuses. Les $V \mathcal{E}$ participent à cette communication intercellulaire (Figure 2).

L'injection de gVE ou de pVE plasmatiques isolées de patients souffrant de SMet (VE SMet) à des souris favorise la dysfonction endothéliale $[4,5]$. Que ce soit avec des gVE ou des $p V \varepsilon$, les altérations vasculaires observées se caractérisent par une baisse de la production de monoxyde d'azote (NO), puissant vasodilatateur, et une production accrue d'espèces réactives de l'oxygène ( $\varepsilon R 0)$ pro-inflammatoires. Les gVE SMet sont spécifiquement enrichies en Rapl (Ras-related protein 1), une petite GTPase liée à différents facteurs de risque cardiovasculaire [22]. Ces gVE-Rap I favorisent les processus d'inflammation et de remodelage vasculaire et s'accumulent dans les plaques athéromateuses [22]. Les pVE SMet sont, elles, enrichies en LPS (lipopolysaccharide), compararativement aux pVE plasmatiques de sujets sains [5]. La neutralisation du récepteur TLR4 réduit la production d'ERO induite par ces pVE-LPS', révélant ainsi un mécanisme différent de celui des gVE SMet dans la mise en place de la dysfonction endothéliale.

L'environnement physiopathologique contribue à favoriser la production de VE délétères en terme métabolique. Ainsi, l'hyperglycémie ou encore l'élévation de l'hormone hypertensive angiotensine-II favorisent la sécrétion de $V \varepsilon$ endothéliales aux propriétés pro-coagulantes, pro-inflammatoires et pro-oxydantes $[23,24]$. L'injection de ces $V \varepsilon$ endothéliales à des rongeurs induit une altération de la réactivité vasculaire, le développement de plaques d'athérome, l'infiltration par des macrophages et la production d'ERO [24]. Différentes études mettent en évidence la modulation négative de facteurs pro-angiogéniques, comme le VEGF ou le miR-126, qui contribuent normalement à la réparation endothéliale dans des conditions physiologiques [23].

Ces échanges d'informations via les $V \varepsilon$ au sein du mur vasculaire se font de manière multilatérale, puisque les $V \varepsilon$ dérivées des cellules dendritiques, des macrophages ou des cellules spumeuses, modulent également la fonction endothéliale en amplifiant la réponse inflammatoire via l'activation de la voie $\mathrm{NF}-\kappa \mathrm{B}$, la production de signaux pro-inflammatoires et l'expression de molécules d'adhérence [25]. Les VE produites par des adipocytes hypoxiés et inflammatoires favorisent, quant à elles, l'attachement des leucocytes aux cellules endothéliales vasculaires, suggérant leur participation dans le processus d'athérosclérose [26]. Enfin, des VE adipocytaires produites dans un environnement hyperglycémique et hyperlipémique, exacerbent les lésions d'ischémie/reperfusion du myocarde chez des souris diabétiques [27]. Ainsi, les VE adipocytaires (produites dans un contexte obésogène) pourraient constituer le lien moléculaire sous-tendant les effets délétères d'une hypertrophie des tissus adipeux périvasculaires et/ou péricardiques et le développement des maladies cardiovasculaires.

\section{Les $V \varepsilon$ et hépatopathies dysmétaboliques}

La stéatopathie non alcoolique (NAFLD) est considérée comme la manifestation hépatique du SMet et se caractérise par une accumulation anormale de graisse intrahépatique en l'absence de consommation excessive d'alcool. Cette stéatose isolée peut évoluer vers une forme plus agressive se caractérisant par une stéatose avec inflammation définissant la stéatohépatite non alcoolique (NASH), qui peut évoluer vers la cirrhose et ses complications (insuffisance hépatique, hépatocarcinome).

La sécrétion de $V \varepsilon$ hépatiques participe à la pathogenèse de ces hépatopathies dysmétaboliques en favorisant les processus inflammatoires, immunitaires, fibrotiques et angiogéniques [8] (Figure 2). Ainsi, l'enrichissement des pVE dérivées d'hépatocytes stéatosiques en facteurs pro-inflammatoires [28] ou en lipides lipotoxiques (céramides ou sphingosine-1-phosphate) [29] contribuent à la chémoattraction des macrophages vers le foie. La présence de certains miR, comme le miR192, contribue, en outre, à l'activation des macrophages [30]. L'accumulation de lipides dans le foie stimule la sécrétion de $p V \varepsilon$ hépatocytaires enrichies en vanine-1 (VNN1), une enzyme dotée d'une activité pro-fibrosante [31]. La VVN1 vésiculaire induit la migration des cellules endothéliales et la formation de tubes vasculaires, deux processus nécessaires à l'angiogenèse pathologique dans le foie, résultant de la NASH.

L'internalisation de VE dérivées d'hépatocytes stéatosiques par les cellules étoilées du foie (HSC) quiescentes conduit à leur activation - un processus critique dans le développement de la fibrose associée aux hépatopathies dysmétaboliques. Ce phénomène fait suite au transfert de miR-128-3p, conduisant à l'inhibition de l'expression de PPAR- $\gamma$ (peroxisome proliferator-acti- 


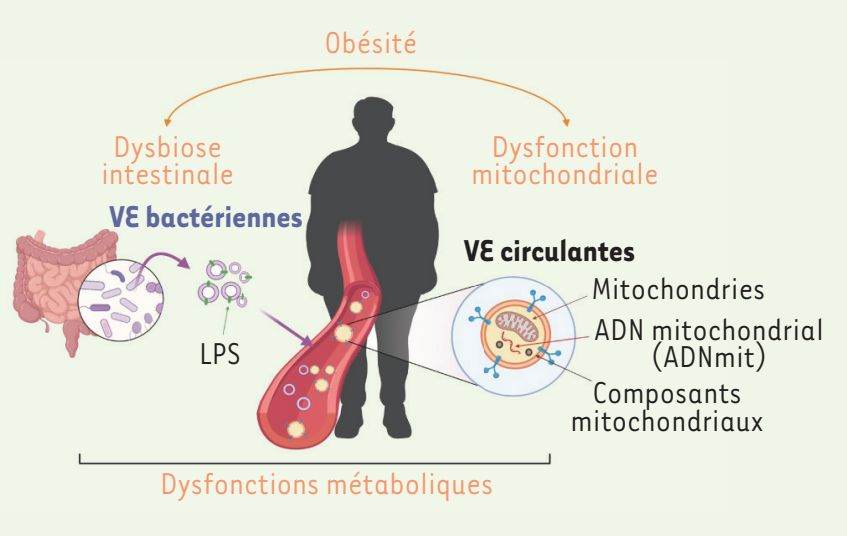

Figure 3. Le transport vésiculaire de mitochondries ou de composés bactériens participe à la mise en place des dysfonctions métaboliques. Les complications métaboliques de l'obésité sont associées à une altération du microbiote intestinal (ou dysbiose), conduisant à la production de VE bactériennes, reflet de cette dysbiose. La dysfonction mitochondriale, souvent associée aux maladies métaboliques, se traduit également par l'export cellulaire, via les $V \varepsilon$, de mitochondries ou de constituants mitochondriaux. Le trafic de cet ensemble de VE à l'échelle de l'organisme participe au développement de dysfonctions métaboliques.

vated receptor $\gamma)$ normalement chargé de maintenir les HSC dans un état quiescent [32]. D'autres miR, tels que le miR-122 et le miR-192, sont également impliqués dans les effets inflammatoires et fibrosants des pVE hépatocytaires produites dans des conditions lipotoxiques [8]. Des corrélations positives entre ces miR, les enzymes hépatiques et la gravité de la maladie hépatique suggèrent que la signature de ces miR vésiculaires pourrait être utilisée comme biomarqueur de l'atteinte hépatique.

Au-delà de leurs effets sur le foie, les VE hépatocytaires pourraient également participer à un dialogue inter-organes contribuant à favoriser les dysfonctions métaboliques. Ainsi, les pVE dérivées d'hépatocytes stéatosiques favorisent l'inflammation et atténuent l'athérosclérose de souris déficientes en ApoE (apolipoprotéine $\varepsilon$ ), suggérant un couplage entre hépatopathies dysmétaboliques et maladies cardiovasculaires dépendant des pVદ [33]. Les pVદ dérivées d'hépatocytes primaires isolés de souris obèses favorisent le stockage lipidique adipocytaire [34]. Enfin, l'hypertrophie adipocytaire conduit à la libération de $V \varepsilon$ adipocytaires qui favorisent la fibrose et la résistance à l'insuline dans le foie [20].

Le dialogue vésiculaire dans un contexte d'obésité pourrait donc s'auto-amplifier, contribuant à entretenir les effets métaboliques délétères via les $V \varepsilon$ et à favoriser le développement de complications métaboliques.

\section{Nouvelles pistes de recherche et perspectives}

\section{Les VE : des vecteurs de mitochondries}

Les mitochondries sont des organites spécifiques des cellules eucaryotes assurant la respiration cellulaire et la production d'ATP; elles jouent donc un rôle majeur dans le métabolisme énergétique. Leur particularité est de posséder leur propre génome sous la forme d'un ADN circulaire (ou ADN mitochondrial, ADNmit), transmis uniquement par la mère et distinct de l'ADN nucléaire. La détection de mitochondries fonctionnelles dans des gVE circulantes a formellement démontré la capacité des $V \varepsilon$ à transporter des organites entiers [35]. Bien que la taille des pVE soit difficilement compatible avec l'encapsulation de mitochondries entières, plusieurs travaux révèlent leur enrichissement en enzymes, lipides (cardiolipines) et ADN mitochondriaux [36, 37].

De récents travaux ont montré une forte augmentation d'ADNmit dans le plasma de patients, ou de souris, souffrant de la NASH [38]. La majorité de cet ADNmit est encapsulé dans des gVE d'origine hépatocytaire, et peut activer le récepteur TLR9, une voie de signalisation impliquée dans le contrôle du développement de la NASH. Le ciblage de l'activation de TLR9 via I'ADN mitochondrial vésiculaire pourrait donc constituer une perspective thérapeutique pour le traitement de cette maladie.

Le transfert de mitochondries via des gVE monocytaires pourrait également participer à la pathogenèse de l'athérosclérose (Figure 3). Le traitement de monocytes par du lipopolysaccharide (LPS) bactérien induit la sécrétion de gVE enrichies en mitochondries dysfonctionnelles, auxquelles sont associés du TNF- $\alpha$ ou de I'ARN mitochondrial oxydé, capables d'induire une réponse pro-inflammatoire dans les cellules endothéliales [39]. Une récente étude suggère que cette excrétion mitochondriale serait couplée à un processus d'autophagie [40]. Les auteurs de cette étude ont également montré que les gVE secrétées par les cardiomyocytes sont phagocytées par les macrophages, phénomène amplifié lors d'un stress cardiaque, révélant un nouveau processus par lequel les cellules immunitaires résidentes du cœur participent à l'homéostasie du tissu cardiaque.

Enfin, plusieurs études ont rapporté des effets protecteurs et immunorégulateurs faisant suite au transfert intercellulaire de mitochondries par les $V \varepsilon$. Une étude récente a ainsi mis en évidence un transfert de mitochondries via les $V \varepsilon$ entre des adipocytes et des macrophages résidents du tissu adipeux [41]. Ce transfert participerait à I'homéostasie immunométabolique de ce tissu, mais serait altéré dans un contexte d'obésité. En outre, des adipocytes «stressés», comme c'est le cas à la suite d'un stress oxydant consécutif à une dysfonction mitochondriale, induit la sécrétion de pVE enrichies 
en mitochondries dysfonctionnelles capables de diffuser dans la circulation et d'être captées par les cardiomyocytes [42]. II en résulte l'activation d'une réponse compensatrice anti-oxydante par le cœur qui protège les cardiomyocytes du stress oxydant aigu, conformément à un paradigme de préconditionnement s'apparentant au concept « d'hormèse ${ }^{1}$. Les mitochondries transférées par des VE dérivées de cellules souches mésenchymateuses (MSC) vont également contribuer à un remodelage tissulaire bénéfique. L'injection de telles $V \varepsilon$ dans des souris utilisées comme modèle murin de défaillance rénale aiguë atténue ainsi la dysfonction rénale et la réponse inflammatoire suite au transfert vésiculaire d'ADNmit et d'ARNm codant un facteur mitochondrial dans les tubules rénaux [43]. À noter que des VE dérivées de cellules souches pluripotentes différenciées en cardiomyocytes, injectées dans le cœur, sont également capables de transférer des cargos mitochondriaux favorisant la biogenèse mitochondriale et la récupération énergétique du cœur après infarctus du myocarde chez des souris [44].

Ces résultats ouvrent donc la voie à des thérapies innovantes fondées sur le transfert spécifique de composés mitochondriaux ou de mitochondries, dans le traitement de maladies présentant une dysfonction mitochondriale.

\section{Les Vદ dérivées du microbiote intestinal : une piste bactérienne à explorer}

Les maladies métaboliques associées à l'obésité sont reliées à des modifications du nombre et de la diversité bactérienne, un phénomène appelé dysbiose. L'avènement de la métagénomique a permis d'identifier des microbiotes obésogène et diabétogène spécifiques de différentes maladies métaboliques. L'altération de l'intégrité de la paroi intestinale, associée au SMet, favorise la translocation de molécules bactériennes, comme le LPS, hautement inflammatoires, ce qui conduit à une endotoxémie métabolique. L'observation concomitante de bactéries dans les fèces de patients et de VE bactériennes présentes dans leur sérum ou leurs urines, illustre la capacité de ces VE, qui dérivent du microbiote intestinal, à traverser la barrière intestinale alors altérée et à se distribuer dans différents fluides de l'organisme [45]. De surcroît, comparées à des pVE plasmatiques de sujets sains, les pVE SMet sont enrichies en LPS ; elles pourraient ainsi signer l'endotoxémie métabolique que l'on retrouve chez les patients souffrant de SMet [5].

Plusieurs études précliniques suggèrent le rôle de médiateurs métaboliques exercé par les $V \varepsilon$ bactériennes dans le développement des dysfonctions métaboliques (Figure 3). L'ingestion de VE bactériennes, isolées de fèces de souris sous régime gras, par des souris saines conduit à leur biodistribution dans plusieurs tissus métaboliques et au développement d'une insulinorésistance [46]. Des analyses métagénomiques de $V \varepsilon$ dérivées du microbiote de souris obèses, révèlent un enrichissement en VE dérivées de bacté-

1 L'hormèse est un processus par lequel un organisme, à qui l'on administre de faibles doses d'agents générateurs de stress, se trouve renforcé et montre ensuite une plus grande résilience à des doses plus élevées de ces mêmes facteurs de stress. ries Pseudomonas panacis (phylum Proteobacteria) qui, à elles seules, peuvent induire une résistance à l'insuline lorsqu'elles sont administrées à des souris saines. De même, des VE dérivées de Porphyromonas gingivalis ${ }^{2}$ transportent des protéases, parmi lesquelles des gingipaïnes, des enzymes associées aux parodontites, qui se retrouvent dans le foie des souris où elles modulent la sensibilité à l'insuline et contribuent au développement du DT2 [47]. Dans les cellules avec lesquelles elles interagissent, les VE bactériennes stimulent également plusieurs mécanismes moléculaires qui se traduisent par des réponses inflammatoires. Parmi ces mécanismes, on peut citer leur liaison au récepteur TLR4, exprimé par les cellules de l'immunité innée, qui déclenche une réponse immunitaire et la sécrétion de cytokines pro-inflammatoires [48].

Dans un contexte de dysbiose, il serait intéressant de promouvoir la production de VE dérivées de bactéries bénéfiques, comme Akkermansia muciniphila dont l'abondance est associée à un statut métabolique sain qui se traduit notamment par une meilleure sensibilité à l'insuline [49]. Les VE dérivées de cette bactérie sont à l'origine d'effets bénéfiques, supérieurs à ceux induits par la bactérie elle-même, sur la barrière intestinale, l'inflammation, la balance énergétique ou les paramètres métaboliques globaux [50].

Ces résultats ouvrent donc la voie à de nouvelles perspectives thérapeutiques consistant en un apport exogène de $V \varepsilon$ dérivées de bactéries bénéfiques.

\section{Conclusion}

L'ensemble des données de la littérature révèle les vésicules extracellulaires (VE) comme des actrices importantes de la communication inter-organes, participant au développement de dysfonctions métaboliques en régulant l'inflammation, le remodelage tissulaire, la sensibilité à l'insuline ou l'homéostasie énergétique. Selon leur origine cellulaire et l'environnement physiopathologique, les $V \varepsilon$ vont produire des réponses cellulaires variées dont les processus restent encore largement méconnus. Mieux caractériser les mécanismes régulant la sécrétion de ces $V \varepsilon$, leur biodistribution et leur mode d'action sont autant de champs qui restent à explorer. Malgré l'intérêt certain que ces $V \varepsilon$ suscitent en tant que biomarqueurs diagnostiques ou vecteurs thérapeutiques, plusieurs limites techniques et métho-

\footnotetext{
2 Porphyromonas gingivalis est une espèce de bactéries présentes dans la cavité
} buccale, responsable de gingivite. 
dologiques restent néanmoins à dépasser avant d'envisager leur utilisation clinique. Ces nanovésicules ont donc su mobiliser la communauté scientifique, dont l'intérêt risque d'aller grandissant au vu des nombreuses propriétés métaboliques qu'on leur prête ainsi que des nombreux mécanismes sous-jacents à leur production et à leurs fonctions restant encore à décrypter. $\diamond$

\section{SUMMARY}

\section{Extracellular vesicles and metabolic diseases: Dangerous liaisons}

Extracellular vesicles ( $\varepsilon V s$ ) correspond to a heterogeneous set of membrane nanovesicles secreted in the extracellular medium and circulating in the various fluids of the body. These $\varepsilon V s$ convey biological material (proteins, lipids, nucleic acids) that they can transfer to target cells/tissues thus modulating their response and/or phenotype. The metabolic dysfunctions characterizing metabolic diseases associated with obesity are associated with changes in circulating $\varepsilon V$ concentrations as well as alterations in their content. The growing interest in $E V$ s as new vectors of intercellular communication has led to question about their role in the development of metabolic complications. In this review, we will discuss the literature on circulating $\varepsilon V s$ as potential markers of metabolic diseases and then detail interorgan dialogue based on this $E V$ trafficking underlying the development of related obesity. Finally, we will discuss future avenues of research that will help to better understand the link between $E V$ s and metabolic diseases. $\diamond$

\section{REMERCIEMENTS}

Les auteures remercient le soutien financier de l'Inserm, de la Société francophone du diabète, la fondation Genavie et la Région Pays de la Loire. Les figures ont été créées avec Biorender.com.

\section{LIENS D'INTÉRÊT}

Les auteures déclarent n'avoir aucun lien d'intérêt concernant les données publiées dans cet article.

\section{RÉFÉRENCES}

1. Alberti G, Zimmet P. The IDF consensus worldwide definition of the METABOLIC SYNDROME. International Diabetes Federation 2006.

2. van Niel G, D’Angelo G, Raposo G. Shedding light on the cell biology of extracellular vesicles. Nat Rev Mol Cell Biol $2018 ; 19: 213-28$.

3. Boulanger CM, Loyer X, Rautou PE, Amabile N. Extracellular vesicles in coronary artery disease. Nat Rev Cardiol $2017 ; 14: 259-72$.

4. Agouni A, Lagrue-Lak-Hal AH, Ducluzeau PH, et al. Endothelial dysfunction caused by circulating microparticles from patients with metabolic syndrome. Am J Pathol $2008 ; 173: 1210-9$.

5. Ali S, Malloci M, Safiedeen Z, et al. LPS-enriched small extracellular vesicles from metabolic syndrome patients trigger endothelial dysfunction by activation of TLR4. Metabolism 2021 : 154727.

6. Amosse J, Durcin M, Malloci M, et al. Phenotyping of circulating extracellular vesicles (EVs) in obesity identifies large $\varepsilon V s$ as functional conveyors of Macrophage Migration Inhibitory Factor. Mol Metab 2018 ; 18 : 134-42.

7. Li S, Wei J, Zhang C, et al. Cell-Derived Microparticles in Patients with Type 2 Diabetes Mellitus: a Systematic Review and Meta-Analysis. Cell Physiol Biochem 2016; $39: 2439-50$.

8. Srinivas AN, Suresh D, Santhekadur PK, et al. Extracellular Vesicles as Inflammatory Drivers in NAFLD. Front Immunol $2020 ; 11: 627424$.

9. Le Lay S, Rome S, Loyer X, Nieto L. Adipocyte-derived extracellular vesicles in health and diseases: Nano-packages with vast biological properties. FASEB BioAdvances $2021 ; 00: 1-13$.
10. Freeman DW, Noren Hooten N, Eitan $\varepsilon$, et al. Altered Extracellular Vesicle Concentration, Cargo and Function in Diabetes Mellitus. Diabetes 2018 ; 67 : 2377-88.

11. Boulanger $C M$, Scoazec $A$, Ebrahimian $T$, et al. Circulating microparticles from patients with myocardial infarction cause endothelial dysfunction. Circulation $2001 ; 104: 2649-52$.

12. Sarlon-Bartoli G, Bennis Y, Lacroix R, et al. Plasmatic level of leukocytederived microparticles is associated with unstable plaque in asymptomatic patients with high-grade carotid stenosis. J Am Coll Cardiol 2013 ; 62 : 143641.

13. Sinning JM, Losch J, Walenta $K$, et al. Circulating CD31+/Annexin V+ microparticles correlate with cardiovascular outcomes. Eur Heart J 2011 ; $2011 ; 32$ : 2034-41.

14. Oshikawa S, Sonoda H, Ikeda M. Aquaporins in Urinary Extracellular Vesicles (Exosomes). Int J Mol Sci $2016 ; 17$.

15. Santamaria-Martos F, Benitez ID, Latorre J, et al. Comparative and functional analysis of plasma membrane-derived extracellular vesicles from obese vs. nonobese women. Clin Nutr 2020 ; 39 : 1067-76.

16. Durcin M, Fleury A, Taillebois $\varepsilon$, et al. Characterisation of adipocyte-derived extracellular vesicle subtypes identifies distinct protein and lipid signatures for large and small extracellular vesicles. J Extracell Vesicles 2017 ; 6 : 1305677.

17. Rome S, Blandin A, Le Lay S. Adipocyte-Derived Extracellular Vesicles: State of the Art. Int J Mol Sci $2021 ; 22$.

18. Deng ZB, Poliakov A, Hardy RW, et al. Adipose tissue exosome-like vesicles mediate activation of macrophage-induced insulin resistance. Diabetes $2009 ; 58: 2498-505$.

19. Ying W, Riopel M, Bandyopadhyay G, et al. Adipose Tissue MacrophageDerived Exosomal miRNAs Can Modulate In Vivo and In Vitro Insulin Sensitivity. Cell 2017 ; 171 : 372-84 el2.

20. Kranendonk ME, Visseren FL, van Balkom BW, et al. Human adipocyte extracellular vesicles in reciprocal signaling between adipocytes and macrophages. Obesity (Silver Spring) 2014 ; 22 : 1296-308.

21. Aswad H, Forterre A, Wiklander OP, et al. Exosomes participate in the alteration of muscle homeostasis during lipid-induced insulin resistance in mice. Diabetologia $2014 ; 57: 2155-64$.

22. Perdomo L, Vidal-Gomez X, Soleti R, et al. Large Extracellular VesicleAssociated Rapl Accumulates in Atherosclerotic Plaques, Correlates With Vascular Risks and Is Involved in Atherosclerosis. Circ Res 2020 ; 127 : 747-60.

23. Jansen $F$, Yang X, Franklin BS, et al. High glucose condition increases NADPH oxidase activity in endothelial microparticles that promote vascular inflammation. Cardiovasc Res ; 98 : 94-106.

24. Burger D, Montezano AC, Nishigaki N, et al. Endothelial microparticle formation by angiotensin II is mediated via Ang II receptor type I/NADPH oxidase/ Rho kinase pathways targeted to lipid rafts. Arterioscler Thromb Vasc Biol 2011 ; 31 : 1898-907.

25. Hosseinkhani B, Kuypers S, van den Akker NMS, et al. Extracellular Vesicles Work as a Functional Inflammatory Mediator Between Vascular Endothelial Cells and Immune Cells. Front Immunol 2018 ; 9 : 1789.

26. Wadey RM, Connolly KD, Mathew D, et al. Inflammatory adipocyte-derived extracellular vesicles promote leukocyte attachment to vascular endothelial cells. Atherosclerosis $2019 ; 283: 19-27$.

27. Gan L, Xie D, Liu J, et al. Small Extracellular Microvesicles Mediated Pathological Communications Between Dysfunctional Adipocytes and Cardiomyocytes as a Novel Mechanism Exacerbating Ischemia/Reperfusion Injury in Diabetic Mice. Circulation $2020 ; 141$ : 968-83.

28. Ibrahim SH, Hirsova P, Tomita K, et al. Mixed lineage kinase 3 mediates release of $\mathrm{C}-\mathrm{X}-\mathrm{C}$ motif ligand 10 -bearing chemotactic extracellular vesicles from lipotoxic hepatocytes. Hepatology 2016; 63 : 731-44.

29. Kakazu $\varepsilon$, Mauer AS, Yin M, Malhi H. Hepatocytes release ceramide-enriched pro-inflammatory extracellular vesicles in an IRElalpha-dependent manner. J Lipid Res 2016 ; 57 : 233-45.

30. Liu XL, Pan Q, Cao HX, et al. Lipotoxic Hepatocyte-Derived Exosomal MicroRNA 192-5p Activates Macrophages Through Rictor/Akt/Forkhead Box Transcription Factor 01 Signaling in Nonalcoholic Fatty Liver Disease. Hepatology $2020 ; 72: 454-69$.

31. Povero D, Eguchi A, Niesman IR, et al. Lipid-induced toxicity stimulates hepatocytes to release angiogenic microparticles that require Vanin-1 for uptake by endothelial cells. Sci Signal 2013; 6 : ra88.

32. Povero D, Panera N, Eguchi A, et al. Lipid-induced hepatocyte-derived extracellular vesicles regulate hepatic stellate cell via microRNAs targeting PPAR-gamma. Cell Mol Gastroenterol Hepatol 2015 ; 1 : 646-63 e4. 


\section{RÉFÉRENCES}

33. Jiang $F$, Chen $Q$, Wang $W$, et al. Hepatocyte-derived extracellular vesicles promote endothelial inflammation and atherogenesis via microRNA-1.J Hepatol $2020 ; 72: 156-66$.

34. Zhao $\mathrm{Y}$, Zhao MF, Jiang $\mathrm{S}$, et al. Liver governs adipose remodelling via extracellular vesicles in response to lipid overload. Nat Commun $2020 ; 11: 719$.

35. Al Amir Dache Z, Otandault A, Tanos R, et al. Blood contains circulating cell-free respiratory competent mitochondria. FASEB J 2020 ; $34: 3616-30$.

36. Haraszti RA, Didiot MC, Sapp $\varepsilon$, et al. High-resolution proteomic and lipidomic analysis of exosomes and microvesicles from different cell sources. J Extracell Vesicles $2016 ; 5: 32570$.

37. D’Acunzo P, Perez-Gonzalez R, Kim Y, et al. Mitovesicles are a novel population of extracellular vesicles of mitochondrial origin altered in Down syndrome. Sci Adv $2021 ; 7$.

38. Garcia-Martinez I, Santoro N, Chen Y, et al. Hepatocyte mitochondrial DNA drives nonalcoholic steatohepatitis by activation of TLR9. J Clin Invest 2016; $126: 859-64$

39. Puhm F, Afonyushkin T, Resch U, et al. Mitochondria Are a Subset of Extracellular Vesicles Released by Activated Monocytes and Induce Type I IFN and TNF Responses in Endothelial Cells. Circ Res $2019 ; 125: 43-52$.

40. Nicolas-Avila JA, Lechuga-Vieco AV, Esteban-Martinez L, et al. A Network of Macrophages Supports Mitochondrial Homeostasis in the Heart. Cell 2020 ; 183 : 94-109 e23.

41. Brestoff JR, Wilen CB, Moley JR, et al. Intercellular Mitochondria Transfer to Macrophages Regulates White Adipose Tissue Homeostasis and Is Impaired in Obesity. Cell Metab 2021; 33 : 270-82 e8.

42. Crewe C, Funcke JB, Li S, et al. Extracellular vesicle-based interorgan transport of mitochondria from energetically stressed adipocytes. Cell Metab 2021; 17 : S1550-4131(21)00365-X.

43. Zhao M, Liu S, Wang C, et al. Mesenchymal Stem Cell-Derived Extracellular Vesicles Attenuate Mitochondrial Damage and Inflammation by Stabilizing Mitochondrial DNA. ACS Nano 2021 ; 15 : 1519-38.

44. Ikeda G, Santoso MR, Tada Y, et al. Mitochondria-Rich Extracellular Vesicles From Autologous Stem Cell-Derived Cardiomyocytes Restore Energetics of Ischemic Myocardium. J Am Coll Cardiol $2021 ; 77: 1073-88$.
45. Nah G, Park SC, Kim K, et al. Type-2 Diabetics Reduces Spatial Variation of Microbiome Based on Extracellur Vesicles from Gut Microbes across Human Body. Sci Rep 2019 ; 9 : 20136

46. Choi Y, Kwon Y, Kim DK, et al. Gut microbe-derived extracellular vesicles induce insulin resistance, thereby impairing glucose metabolism in skeletal muscle. Sci Rep $2015 ; 5$ : 15878

47. Seyama M, Yoshida K, Yoshida K, et al. Outer membrane vesicles of Porphyromonas gingivalis attenuate insulin sensitivity by delivering gingipains to the liver. Biochim Biophys Acta Mol Basis Dis 2020 ; 1866 165731.

48. Gilmore WJ, Johnston EL, Zavan L, et al. Immunomodulatory roles and novel applications of bacterial membrane vesicles. Mol Immunol $2021 ; 134$ $72-85$

49. Dao MC, Everard A, Aron-Wisnewsky J, et al. Akkermansia muciniphila and improved metabolic health during a dietary intervention in obesity: relationship with gut microbiome richness and ecology. Gut $2016 ; 65: 426-$ 36.

50. Ashrafian F, Shahriary A, Behrouzi A, et al. Akkermansia muciniphila-Derived Extracellular Vesicles as a Mucosal Delivery Vector for Amelioration of Obesity in Mice. Front Microbiol 2019; $10: 2155$.

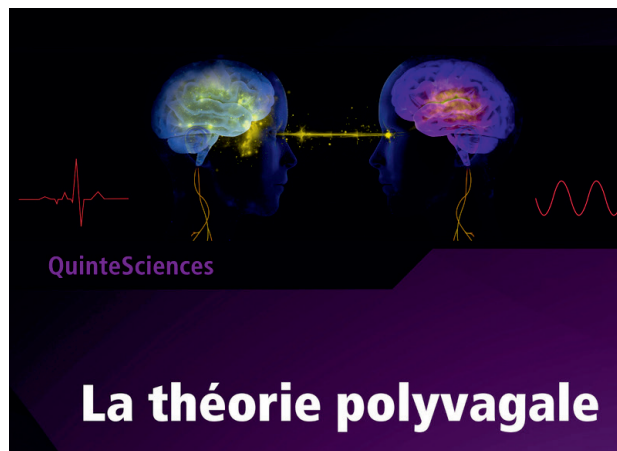

Fondements neurophysiologiques des émotions, de l'attachement, de la communication et de I'autorégulation

\section{Stephen W. Porges}

Iraduit par Nico Milantoni et Isabelle Chosson-Argentier

\section{edpsciences}

\section{Enfin disponible en français}

\section{L'ouvrage de référence de S.W.Porges}

S.W.Porges est le spécialiste mondial du lien unissant le système nerveux autonome au comportement social. Il nous offre de passionnantes perspectives sur la façon dont notre système nerveux autonome gère inconsciemment notre engagement social, la confiance, l'intimité.

La traduction a été réalisée par des experts : Nico Milantoni est psychologue, praticien et formateur de la méthode Hipérion. Isabelle Chosson-Argentier est Docteur en pharmacie, conseil en nutrition, micronutritionniste, phyto- et aromathérapeute, praticienne de la méthode Hipérion.

ISBN : 978-2-7598-2498-4 edpsciences.org 